Check for updates

Cite this: Phys. Chem. Chem. Phys., 2019, 21, 1014

Received 20th November 2018, Accepted 14th December 2018

DOI: $10.1039 / \mathrm{c} 8 \mathrm{cp} 07160 \mathrm{~h}$ rsc.li/pccp

\section{Magnetic order and enhanced exchange in the quasi-one-dimensional molecule-based antiferromagnet $\mathrm{Cu}\left(\mathrm{NO}_{3}\right)_{2}(\mathrm{pyz})_{3} \dagger$}

\author{
Benjamin M. Huddart, (D) *a Jamie Brambleby, ${ }^{b}$ Tom Lancaster, (D) ${ }^{a}$ \\ Paul A. Goddard, (D) ${ }^{\mathrm{b}}$ Fan Xiao, ${ }^{\text {cd }}$ Stephen J. Blundell, (D) e Francis L. Pratt, (D) \\ John Singleton, ${ }^{9}$ Piero Macchi, (D) ${ }^{\text {ch }}$ Rebecca Scatena, (D) ${ }^{c}$ Alyssa M. Barton and \\ Jamie L. Manson (iD *i
}

The quasi-one-dimensional molecule-based Heisenberg antiferromagnet $\mathrm{Cu}\left(\mathrm{NO}_{3}\right)_{2}(\mathrm{pyz})_{3}$ has an intrachain coupling $J=13.7(1) \mathrm{K}$ $\left(\hat{H}=J \sum_{i} S_{i} \cdot S_{i+1}\right)$ and exhibits a state of long-range magnetic order below $T_{\mathrm{N}}^{i}=0.105(1) \mathrm{K}$. The ratio of interchain to intrachain coupling is estimated to be $\left|J^{\prime}\right| J \mid=3.3 \times 10^{-3}$, demonstrating a high degree of isolation for the $\mathrm{Cu}$ chains.

Recent progress in the field of molecular magnetism has shown the clear potential for gaining control over the structural building blocks of molecular materials in order to engineer lowdimensional magnetic properties. In some notable cases, large intermolecular spacing and pseudo Jahn-Teller (JT) distortions result in exchange interactions between transition metal ions being facilitated by bridging ligands such as pyrazine $\left(=\mathrm{pyz}=\mathrm{C}_{4} \mathrm{H}_{4} \mathrm{~N}_{2}\right)$. This allows for the construction of polymeric networks whose primary exchange occurs along one, two or

${ }^{a}$ Centre for Materials Physics, Durham University, South Road, Durham DH1 3LE, UK. E-mail: benjamin.m.huddart@durham.ac.uk

${ }^{b}$ Department of Physics, University of Warwick, Gibbet Hill Road, Coventry, CV4 $7 A L, U K$

${ }^{c}$ Department of Chemistry and Biochemistry, University of Bern, Freiestrasse 3, Bern, 3012, Switzerland

${ }^{d}$ Laboratory for Neutron Scattering and Imaging, Paul Scherrer Institut, CH-5232 Villigen PSI, Switzerland

${ }^{e}$ Department of Physics, Clarendon Laboratory, University of Oxford, Parks Road, Oxford OX1 3PU, UK

${ }^{f}$ ISIS Pulsed Muon Facility, STFC Rutherford Appleton Laboratory, Chilton, Didcot, OX11 OQX, UK

${ }^{g}$ National High Magnetic Field Laboratory, Los Alamos National Laboratory, MS-E536, Los Alamos, New Mexico 87545, USA

${ }^{h}$ Polytechnic of Milan, Department of Chemistry, Materials and Chemical Engineering, via Mancinelli 7 20131, Milan, Italy

${ }^{i}$ Department of Chemistry and Biochemistry, Eastern Washington University,

Cheney, Washington 99004, USA. E-mail: jmanson@ewu.edu

$\dagger$ Electronic supplementary information (ESI) available: $\mathrm{CIF}$ for $\mathrm{Cu}\left(\mathrm{NO}_{3}\right)_{2}(\mathrm{pyz})_{3}$ at $173 \mathrm{~K}$. Experimental details for magnetic susceptibility, pulsed-field magnetization and muon-spin spectroscopy measurements. Additional information about density functional theory calculations. CCDC 1879250. For ESI and crystallographic data in CIF or other electronic format see DOI: 10.1039/c8cp07160h three dimensions. ${ }^{1}$ The manipulation of these building blocks, combined with further ingredients such as counterions ${ }^{2}$ and additional ligands, ${ }^{3}$ enables the synthesis of materials with a wide range of magnetic properties. Furthermore, such systems act as experimental realizations of simple and well-studied model systems, such as the one-dimensional ${ }^{4}$ and two-dimensional ${ }^{5}$ $S=1 / 2$ quantum Heisenberg antiferromagnets.

The molecule-based antiferromagnet $\mathrm{Cu}\left(\mathrm{NO}_{3}\right)_{2}(\mathrm{pyz})$ (1) represents a highly-ideal experimental realization of the one-dimensional quantum Heisenberg antiferromagnet (1DQHAFM), ${ }^{6}$ described by the Hamiltonian $\hat{H}=J \sum_{i} S_{i} \cdot S_{i+1}$. Exchange coupling between $\mathrm{Cu}^{2+}$ ions is facilitated by pyz ligands [see Fig. 1(a)] with an exchange constant $J=10.3(1) \mathrm{K}$ determined from magnetic susceptibility. ${ }^{6}$ Weak interchain coupling $J^{\prime}$ results in a state of 3D long range magnetic order at $T_{\mathrm{N}}=0.107 \mathrm{~K}$, which was detected using muon-spin relaxation. ${ }^{7}$ These measurements give an estimate $\left|J^{\prime}\right| J \mid=$ $4.4 \times 10^{-3}$ for the ratio of interchain to intrachain coupling. ${ }^{7}$

In this paper, we report the magnetic properties of the Heisenberg antiferromagnetic chain system $\mathrm{Cu}\left(\mathrm{NO}_{3}\right)_{2}(\mathrm{pyz})_{3}$ (2). Like 1, 2 comprises Heisenberg $S=1 / 2 \mathrm{Cu}^{2+}$ ions connected via pyz ligands to form a chain along the $a$-axis [Fig. 1(b)]. ${ }^{8}$ Different from 1, each metal centre has two further transcoordinated non-bridging pyz ligands extending perpendicular to the chains, keeping them well separated [Fig. 1(b)]. This results in regular octahedral coordination of the $\mathrm{Cu}^{2+}$ ions in 2 , which has a much higher degree of symmetry than the distorted octahedral environment for $\mathrm{Cu}^{2+}$ in $\mathbf{1}$ [Fig. 1(a)]. For 2, we obtain an intrachain coupling of $J=13.7(1) \mathrm{K}$ (30\% larger than in 1) from measurements of magnetic susceptibility and pulsed-field magnetization and we argue that different local environments for the $\mathrm{Cu}^{2+}$ ions is the main factor responsible for this higher intrachain coupling. Muon-spin spectroscopy reveals a state of long range magnetic order below $T_{\mathrm{N}}=0.105(1) \mathrm{K}$. The ratio of intrachain to interchain coupling is lower than that obtained for 1, suggesting that this system is an even more successful realization of a 1DQHAFM. 
(a)

We prepared deep blue rods of 2 following the procedure previously described by Parkin et al. $\ddagger^{8}$ We re-determined the $\mathrm{X}$-ray crystal structure of $\mathbf{2}$ as it is relevant to our interpretation of its magnetic properties (see ESI $\dagger$ ). Different to 1, which contains four oxygen donor atoms (from chelated nitrate ligands) and two $\mathrm{N}$-donor atoms from pyz, 2 contains two oxygen donor atoms from coordinated $\mathrm{NO}_{3}{ }^{-}$ligands and four $\mathrm{N}$-donor atoms belonging to pyz $[\mathrm{Cu}-\mathrm{N}(1)=2.0719(14)$ and $\mathrm{Cu}-\mathrm{N}(3)=2.0149(16) \AA]$. The local coordination geometry of the $\mathrm{Cu}^{2+}$ ion in 2 is elongated due to a JT distortion along the $\mathrm{O}-\mathrm{Cu}-\mathrm{O}$ axis $[\mathrm{Cu}-\mathrm{O}(1)=2.3808(11) \AA]$. Importantly, this places the magnetic $\mathrm{d}_{x^{2}-y^{2}}$ orbital in the $\mathrm{CuN}_{4}$ plane such that it can interact with two other $\mathrm{Cu}^{2+}$ ions along the chain through the pyz molecules. The resulting structure is that of a polymeric linear chain [Fig. 1(c)] which propagates along the crystallographic $a$-axis. Successive chains stagger along the $c$-direction and pack to form pseudo two-dimensional sheets in the $a b$-plane [Fig. 1(c)]. Supramolecular interactions within these sheets are weak whereas $\mathrm{H}(2)$ forms a bifurcated interaction with the two non-coordinated oxygen atoms from $\mathrm{NO}_{3}{ }^{-}$on an adjacent chain, $\mathrm{O}(2)$ and $\mathrm{O}(3)$, at distances of 2.5959(15) $\AA$ and 2.5548(14) A, respectively. As a result of these weak interchain interactions we anticipate small magnetic coupling between chains as well.

The measured molar susceptibility, $\chi_{\mathrm{mol}}$, of 2 [Fig. 2(a)] shows a broad maximum at $9 \mathrm{~K}$ indicative of the individual
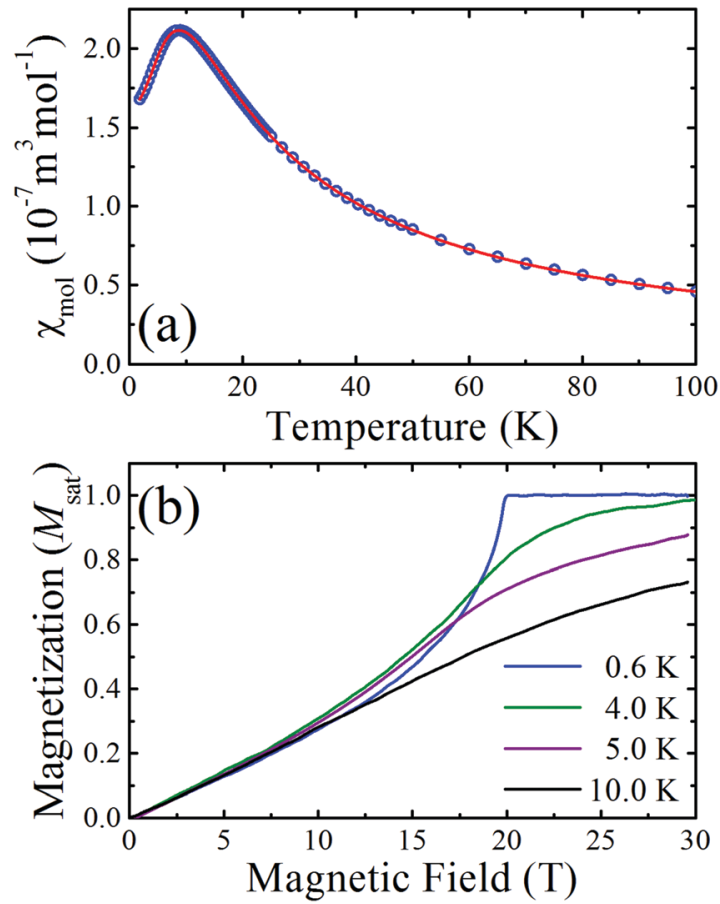

Fig. 2 (a) Molar susceptibility $\left(\chi_{\mathrm{mol}}\right)$ vs. temperature for 2 shows a broad maximum at $9 \mathrm{~K}$. (b) Magnetization in units of the saturation magnetization $\left(M_{\text {sat }}\right)$ vs. magnetic field provided by a short-pulse magnet. Down sweeps of the magnetic field at various temperatures are shown. Data at $0.6 \mathrm{~K}$ are typical of well-isolated $S=1 / 2$ antiferromagnetic chains.

copper moments becoming correlated along the chains and the onset of low-dimensional behaviour below this temperature. Motivated by the structural analysis, the data are fitted to a model of $S=1 / 2$ Heisenberg spins connected in a 1D chain with a single nearest-neighbour interaction $J \dagger^{10}$ The resultant fitted parameters are $g=2.04(1)$ and $J=13.7(1) \mathrm{K}$. A Curie-Weiss analysis of the inverse susceptibility data for temperatures above $100 \mathrm{~K}$ returned fitted values $g=2.03(1)$ and an antiferromagnetic Curie-Weiss temperature $\left|\theta_{\mathrm{cw}}\right|=5.4(5) \mathrm{K}$, which is consistent with the energy scale and nature of the intrachain Heisenberg exchange constant.

Pulsed-field magnetization ${ }^{2}$ measurements up to $60 \mathrm{~T}$ (rise time to full field $\sim 10 \mathrm{~ms}$ ) were carried out at the National High Magnetic Field Laboratory in Los Alamos. The magnetization measured at several temperatures is shown in Fig. 2(b) as a fraction of the saturated value. The concave rise observed at the lowest temperature and the sharp change in gradient at saturation is characteristic of highly isolated $S=1 / 2$ antiferromagnetic chains, where, for the ideal system, $\mathrm{d} M / \mathrm{d} B$ is known to diverge at the saturation field. ${ }^{11}$ As the temperature increases the magnetization exhibits a more gradual approach to saturation.

The structural, susceptibility and magnetization data all imply that the interchain interactions are orders of magnitude smaller than the intrachain exchange $(J)$. In this case, the lowtemperature value of $J$ can be estimated from the saturation field $B_{\mathrm{c}}{ }^{2}$ via the expression $g \mu_{\mathrm{b}} B_{\mathrm{c}}=2 J$. From the $T=0.6 \mathrm{~K}$ magnetization data it is found that $B_{\mathrm{c}}=19.9(1) \mathrm{T}$ and so, using the $g$-factor determined from the Curie-Weiss fit, $J$ is found to 


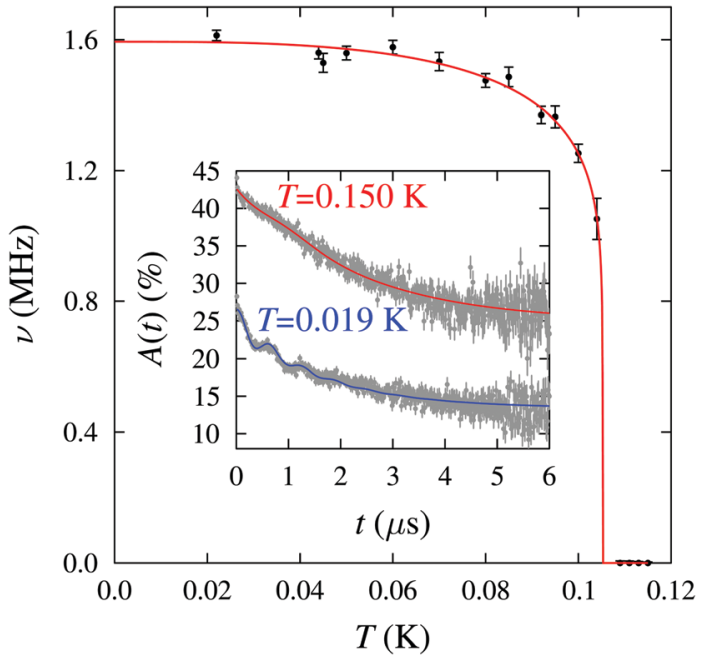

Fig. 3 Temperature dependence of the muon precession frequency $\nu$ for the fits described in the text. Inset: Example $\mu^{+} S R$ spectra for 2 measured above and below the magnetic ordering temperature.

be 13.6(1) $\mathrm{K}$, in agreement with the fits of the magnetic susceptibility.

Implanted muons are very sensitive to small magnetic fields and were therefore used to probe the low-temperature magnetic behaviour. Zero-field muon-spin relaxation $\left(\mathrm{ZF} \mu^{+} \mathrm{SR}\right)$ measurements were made on a polycrystalline sample using the low temperature facility (LTF) spectrometer at the Swiss Muon Source. Example $\mu^{+}$SR spectra are shown in Fig. 3 (inset). For $T<0.105 \mathrm{~K}$ we resolve oscillations at a single frequency in the asymmetry, $A(t)$, characteristic of quasistatic long range magnetic order (LRO) at one magnetically distinct muon site. We note that, in an earlier work on 1, oscillations in the $\mu^{+} \mathrm{SR}$ spectra were observed at two distinct frequencies; ${ }^{7}$ the additional pyz branches in 2 may block muons from occupying one of the candidate muon sites. We fit the spectra to the functional form $A(t)=A_{1} \mathrm{e}^{-\lambda_{1} t}+A_{2} \mathrm{e}^{-\lambda_{2} t} \cos (2 \pi \nu t+\phi)+A_{\mathrm{bg}}$, where the components with amplitudes $A_{1}$ and $A_{2}$, due to muons stopping in the sample, have relaxation rates $\lambda_{1}$ and $\lambda_{2}$ respectively and the component with amplitude $A_{2}$ oscillates with a frequency $\nu$. $A_{\mathrm{bg}}$ is the constant background asymmetry due to muons in the sample holder or those with their spins aligned parallel to the internal field. Above $T=0.105 \mathrm{~K}$ the spectra change shape and we instead fit the data to the sum of a Gaussian and an exponential relaxation. The oscillation frequency $\nu$ (Fig. 3) is related to the magnitude of the magnetic field at the muon site through $\nu=\gamma_{\mu} B / 2 \pi$ where $\gamma_{\mu}$ is the muon gyromagnetic ratio $\left(=2 \pi \times 135.5 \mathrm{MHz} \mathrm{T}^{-1}\right)$ and serves as an effective order parameter for the system. A fit to the phenomenological function $\nu(T)=$ $\nu(0)\left[1-\left(T / T_{\mathrm{N}}\right)^{\alpha}\right]^{\beta}$ with $\alpha=3$ (fixed) yields $\nu(0)=1.59(1) \mathrm{MHz}$, $\beta=0.12(2)$ and $T_{\mathrm{N}}=0.105(1) \mathrm{K}$. A precipitous drop in $\nu$ on approaching $T_{\mathrm{N}}$ and an accompanying small value for the critical exponent $\beta$ is also observed in $\mathbf{1}$, for which $\beta=0.18(5),{ }^{7}$ and likely reflects the reduced dimensionality of these systems.

An empirical formula based on Monte Carlo simulations ${ }^{12}$ allows us to estimate the interchain coupling $J^{\prime}$ from our measurements of $J$ and $T_{\mathrm{N}}$. Substituting $|J| / k_{\mathrm{B}}=13.7 \mathrm{~K}$ and $T_{\mathrm{N}}=0.105(1) \mathrm{K}$ we obtain $\left|J^{\prime}\right| / k_{\mathrm{B}}=0.045 \mathrm{~K}$ and $\left|J^{\prime} J\right|=3.3 \times 10^{-3}$. These values are very similar to those obtained for $1 .{ }^{7}$ Furthermore, we can obtain an estimate of the magnetic moment of $\mathrm{Cu}^{2+}$ in this system through $\left.{ }^{13} m \approx 1.017\left|J^{\prime}\right| J\right|^{1 / 2}$ and obtain $m \approx 0.059 \mu_{\mathrm{B}}$. The moment is very small, highlighting the effect of quantum fluctuations in suppressing the ordered moment in this lowdimensional system. It is worth noting that the ratio of larger muon precession frequency observed for $\mathbf{1}$ and the corresponding frequency for 2 is similar to the ratio of the $\mathrm{Cu}^{2+}$ magnetic moments in these two systems. This suggests that the muon site in $\mathbf{2}$ is similar to the higher frequency site in $\mathbf{1}$, but with a smaller local field as a result of the slightly smaller $\mathrm{Cu}^{2+}$ moment.

Periodic DFT Calculations were carried out using the CRYSTAL14 software. ${ }^{14}$ The functional B3LYP was used with basis set $86-411 \mathrm{G}(41 \mathrm{~d})^{15}$ for the $\mathrm{Cu}$ and pob-TZVP ${ }^{16}$ for all the other atoms. Gas-phase DFT calculations on a dimeric unit were carried out with Gaussian $09^{17}$ using again the B3LYP functional and the basis set $6-311 \mathrm{G}(2 \mathrm{~d}, 2 \mathrm{p})$. We have calculated the exchange couplings $J$ using both periodic DFT and gas phase DFT on a single dimer and find $J=14.88 \mathrm{~K}$ and $J=11.49 \mathrm{~K}$ respectively. Our calculations indicate antiferromagnetic coupling between $\mathrm{Cu}$ spins and show reasonable agreement with the values of $J$ obtained experimentally. These values are larger than the coupling constants $J=10.70 \mathrm{~K}$ and $J=11.02 \mathrm{~K}$ obtained for $1 \mathrm{using}$ periodic DFT and a dimer fragment approach repectively, ${ }^{9}$ supporting the experimentally observed trend. The calculated spin density distribution through the pyz ligand is shown in Fig. 4. The largest part of the spin density is located at the $\mathrm{Cu}$ atoms, with some delocalization to the adjacent $\mathrm{N}$ atoms.

Considering the DFT results, we can rationalize the computed $J$ in terms of symmetry, orbital overlap and structural comparisons. In both 1 and 2, a JT distortion places lobes of the magnetic $\mathrm{Cu}$ $\mathrm{d}_{x^{2}-y^{2}}$ orbitals along the $\mathrm{Cu}-\mathrm{N}$ bonds. The primary exchange pathway between $\mathrm{Cu}^{2+}$ ions is through the bridging pyz ligands along the $1 \mathrm{D}$ chain resulting in similar magnetic properties for $\mathbf{1}$ and 2. However, the enhanced magnetic exchange observed in 2 requires careful analyses of the local environment surrounding the $\mathrm{Cu}^{2+}$ ions. Aside from the shared inversion symmetry of the $\mathrm{Cu}^{2+}$ ion in $\mathbf{1}$ and 2 , the enhanced spin exchange in 2 results

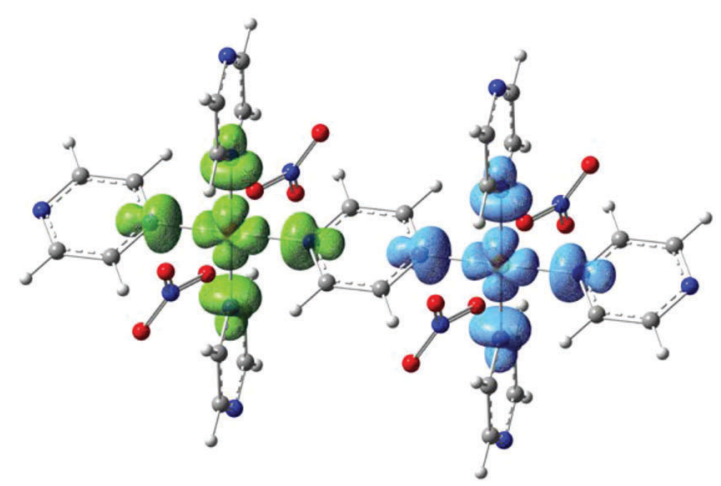

Fig. 4 Spin density distribution through the pyrazine ligand in $\mathbf{2}$ calculated using density functional theory. 
from the particular bonds and angles making up the coordination sphere. In 1 the $d_{x^{2}-y^{2}}$ orbital lies in the plane containing the $\mathrm{Cu}-\mathrm{N}$ bonds $(=1.976 \AA$ ) and the two shorter $\mathrm{Cu}-\mathrm{O}(1)$ bonds $(=2.002 \AA)$. Nitrate coordination in $\mathbf{1}$ is quite different wherein it chelates to the $\mathrm{Cu}^{2+}$ ion resulting in a $\mathrm{O}(1)-\mathrm{Cu}-\mathrm{O}(2)$ bite angle of $56.3^{\circ}$, far from the ideal $90^{\circ}$ observed for purely octahedral symmetry [see Fig. 1(a)]. For 2, the $\mathrm{d}_{x^{2}-y^{2}}$ orbital lies in the plane containing four $\mathrm{Cu}-\mathrm{N}$ bonds with the $\mathrm{Cu}-\mathrm{O}$ bonds lying perpendicular to this plane [see Fig. 1(b)]. The net result is a higher symmetry environment for the $\mathrm{Cu}^{2+}$ in 2 relative to 1 . In terms of the electronic structure, the lower symmetry environment of $\mathrm{Cu}$ in $\mathbf{1}$ can be expected to result in splittings of the $\mathrm{d}$ orbitals, altering the energy differences between the various $\mathrm{Cu}$ $\mathrm{d}$ orbitals and the $\mathrm{sp}^{2}$-hybridized orbital at $\mathrm{N}$ responsible for superexchange. For example, we expect the repulsion between $\mathrm{O}(2)$ and the magnetic $\mathrm{Cu} \mathrm{d}_{x^{2}-y^{2}}$ orbital (which would be smaller if $\mathrm{O}(2)$ was occupying the ideal octahedral position) to raise the energy of electrons in the $\mathrm{d}_{x^{2}-y^{2}}$ orbital, thus reducing the contribution of this superexchange pathway to $J$.

In 1 and 2 the angles between pyz and the plane containing the $\mathrm{d}_{x^{2}-y^{2}}$ orbitals are similar $\left(51^{\circ}\right.$ and $55^{\circ}$ respectively) and we find that $J$ in $\mathbf{1}$ is smaller despite the tilt angle being closer to the theoetically ideal value of $45^{\circ}$ which would be expected to maximize $\pi$-overlap. ${ }^{18}$ This is consistent with an X-ray charge-density study on 1 and the 3D coordination polymer $\left[\mathrm{Cu}\left(\mathrm{NO}_{3}\right)(\mathrm{pyz})_{2}\right] \mathrm{NO}_{3} \cdot \mathrm{H}_{2} \mathrm{O}(3){ }^{9}$ Bond ellipticity profiles ruled out $\pi$-type exchange between the $\mathrm{Cu}$ $\mathrm{d}_{y z}$ and $\mathrm{d}_{x y}$ orbitals and the pyz $\pi$ orbitals, favouring a $\sigma$-only exchange mechanism. This places emphasis on the $\mathrm{Cu}-\mathrm{N}$ interactions and these are most important factor in driving the topology of the spin density.

Finally, $\mathbf{3}$ is structurally similar to $\mathbf{1}$ and $\mathbf{2}$, with pyz ligands providing the primary exchange pathway between $\mathrm{Cu}^{2+}$ ions. ${ }^{9}$ The intrachain $\mathrm{Cu}-\mathrm{N}$ bonds and the $\mathrm{Cu}-\mathrm{N}$ bonds of the terminal pyz ligands in 2 are both shorter than the $\mathrm{Cu}-\mathrm{N}$ bonds in $3 .^{9}$ The shorter intrachain $\mathrm{Cu}-\mathrm{N}$ bonds in 2 concentrate electron density along these bonds, enhancing exchange along the chain. By analogy, the shorter apical $\mathrm{Cu}-\mathrm{O}$ bond in 3 (2.337 $\mathrm{A})$ than in $2(2.381 \AA)$ results in a greater depletion of the spin density in the $\mathrm{CuN}_{4}$ plane, reducing the effectiveness of the magnetic exchange through pyz. The magnitude of $J$ in $\mathbf{1}$ is intermediate between 2 and 3 owing to ligand variation in the $\mathrm{Cu}$ equatorial plane. Oxygen is more electronegative than $\mathrm{N}$ and this likely plays a role in depleting spin density away from the intrachain $\mathrm{Cu}-\mathrm{N}$ bond axis in $\mathbf{1}$.

In conclusion, the spin- $1 / 2$ chain compound $\mathrm{Cu}\left(\mathrm{NO}_{3}\right)_{2}(\mathrm{pyz})_{3}$ exhibits a larger intrachain coupling $J=13.7 \mathrm{~K}$ and a lower magnetic ordering temperature $T_{\mathrm{N}}=0.105 \mathrm{~K}$ than $\mathrm{Cu}\left(\mathrm{NO}_{3}\right)_{2}(\mathrm{pyz})$, making it a more successful realization of a 1DQHAFM. The slightly enhanced $J$ is likely due to the details of the local $\mathrm{Cu}^{2+}$ environment, while effective chain isolation is maintained by non-bridging pyz branches.

\section{Conflicts of interest}

There are no conflicts to declare.

\section{Acknowledgements}

We are grateful to the Swiss Muon Source and thank Chris Baines for experimental assistance. We thank EPSRC for financial support under grants EP/N024028/1, EP/N024486/1 and EP/ N023803/1. BMH thanks STFC for support via a studentship. The work at EWU was supported by the U.S. National Science Foundation (NSF) under grant no. DMR-1703003. PAG acknowledges that this project has received funding from the European Research Council (ERC) under the European Union's Horizon 2020 research and innovation programme (Grant Agreement No. 681260). Part of this work was carried out at the National High Magnetic Field Laboratory, which is funded by NSF Cooperative Agreement DMR-1157490, the State of Florida and U.S. DoE. It was also supported by the US DoE Basic Energy Science Field Work Project Science in 100 T. JS thanks the University of Oxford for the provision of a Visiting Professorship. PM and RS acknowledge funding from the Swiss National Science Foundation under grant no. 160157. Data presented in this paper will be made available via DOI: 10.15128/r23b5918582.

\section{Notes and references}

$\ddagger$ Further increasing the amount of pyrazine in the chemical reaction yields square plates of $\left[\mathrm{Cu}\left(\mathrm{NO}_{3}\right)(\mathrm{pyz})_{2}\right] \mathrm{NO}_{3} \cdot \mathrm{H}_{2} \mathrm{O}$ recently reported by some of us. ${ }^{9}$

1 J. L. Manson, J. A. Schlueter, K. A. Funk, H. I. Southerland, B. Twamley, T. Lancaster, S. J. Blundell, P. J. Baker, F. L. Pratt, J. Singleton, R. D. McDonald, P. A. Goddard, P. Sengupta, C. D. Batista, L. Ding, C. Lee, M.-H. Whangbo, I. Franke, S. Cox, C. Baines and D. Trial, J. Am. Chem. Soc., 2009, 131, 6733-6747.

2 P. A. Goddard, J. Singleton, P. Sengupta, R. D. McDonald, T. Lancaster, S. J. Blundell, F. L. Pratt, S. Cox, N. Harrison, J. L. Manson, H. I. Southerland and J. A. Schlueter, New J. Phys., 2008, 10, 083025.

3 P. A. Goddard, J. Singleton, I. Franke, J. S. Möller, T. Lancaster, A. J. Steele, C. V. Topping, S. J. Blundell, F. L. Pratt, C. Baines, J. Bendix, R. D. McDonald, J. Brambleby, M. R. Lees, S. H. Lapidus, P. W. Stephens, B. W. Twamley, M. M. Conner, K. Funk, J. F. Corbey, H. E. Tran, J. A. Schlueter and J. L. Manson, Phys. Rev. B, 2016, 93, 094430.

4 G. Müller, H. Thomas, H. Beck and J. C. Bonner, Phys. Rev. B: Condens. Matter Mater. Phys., 1981, 24, 1429-1467.

5 E. Manousakis, Rev. Mod. Phys., 1991, 63, 1-62.

6 P. R. Hammar, M. B. Stone, D. H. Reich, C. Broholm, P. J. Gibson, M. M. Turnbull, C. P. Landee and M. Oshikawa, Phys. Rev. B: Condens. Matter Mater. Phys., 1999, 59, 1008-1015.

7 T. Lancaster, S. J. Blundell, M. L. Brooks, P. J. Baker, F. L. Pratt, J. L. Manson, C. P. Landee and C. Baines, Phys. Rev. B: Condens. Matter Mater. Phys., 2006, 73, 020410.

8 T. Otieno, A. M. Gipson and S. Parkin, J. Chem. Crystallogr., 2002, 32, 81-85.

9 L. H. R. Dos Santos, A. Lanza, A. M. Barton, J. Brambleby, W. J. A. Blackmore, P. A. Goddard, F. Xiao, R. C. Williams, T. Lancaster, F. L. Pratt, S. J. Blundell, J. Singleton, J. L. Manson and P. Macchi, J. Am. Chem. Soc., 2016, 138, 2280-2291. 
10 D. C. Johnston, R. K. Kremer, M. Troyer, X. Wang, A. Klümper, S. L. Bud'ko, A. F. Panchula and P. C. Canfield, Phys. Rev. B: Condens. Matter Mater. Phys., 2000, 61, 9558-9606.

11 J. C. Bonner and M. E. Fisher, Phys. Rev., 1964, 135, A640-A658.

12 C. Yasuda, S. Todo, K. Hukushima, F. Alet, M. Keller, M. Troyer and H. Takayama, Phys. Rev. Lett., 2005, 94, 217201.

13 H. J. Schulz, Phys. Rev. Lett., 1996, 77, 2790-2793.

14 R. Dovesi, R. Orlando, A. Erba, C. M. Zicovich-Wilson, B. Civalleri, S. Casassa, L. Maschio, M. Ferrabone, M. De La Pierre, P. D’Arco, Y. Noël, M. Causà, M. Rérat and B. Kirtman, Int. J. Quantum Chem., 2014, 114, 1287-1317.

15 K. Doll and N. Harrison, Chem. Phys. Lett., 2000, 317, 282-289.

16 M. F. Peintinger, D. V. Oliveira and T. Bredow, J. Comput. Chem., 2013, 34, 451-459.

17 M. J. Frisch, G. W. Trucks, H. B. Schlegel, G. E. Scuseria, M. A. Robb, J. R. Cheeseman, G. Scalmani, V. Barone,
B. Mennucci, G. A. Petersson, H. Nakatsuji, M. Caricato, X. Li, H. P. Hratchian, A. F. Izmaylov, J. Bloino, G. Zheng, J. L. Sonnenberg, M. Hada, M. Ehara, K. Toyota, R. Fukuda, J. Hasegawa, M. Ishida, T. Nakajima, Y. Honda, O. Kitao, H. Nakai, T. Vreven, J. A. Montgomery, Jr., J. E. Peralta, F. Ogliaro, M. Bearpark, J. J. Heyd, E. Brothers, K. N. Kudin, V. N. Staroverov, R. Kobayashi, J. Normand, K. Raghavachari, A. Rendell, J. C. Burant, S. S. Iyengar, J. Tomasi, M. Cossi, N. Rega, J. M. Millam, M. Klene, J. E. Knox, J. B. Cross, V. Bakken, C. Adamo, J. Jaramillo, R. Gomperts, R. E. Stratmann, O. Yazyev, A. J. Austin, R. Cammi, C. Pomelli, J. W. Ochterski, R. L. Martin, K. Morokuma, V. G. Zakrzewski, G. A. Voth, P. Salvador, J. J. Dannenberg, S. Dapprich, A. D. Daniels, Farkas, J. B. Foresman, J. V. Ortiz, J. Cioslowski and D. J. Fox, Gaussian 09, Gaussian Inc., Wallingford CT, 2009.

18 H. W. Richardson and W. E. Hatfield, J. Am. Chem. Soc., 1976, 98, 835-839. 